\title{
XIV.
}

Aus der chirurgischen Abtheilung des Herrn Geheimrath Prof. Dr. Hahn im städtischen Krankenhause im Friedrichshain zu Berlin.

\section{Ueber Strumitis nach Pneumonie im Anschluss an einen Fall von Strumitis purulenta mit Mischinfection.}

\author{
Von \\ Dr. Schlender, \\ Oberarzt im Infanterie-Regiment Nr. 138, commandirt zur Klinik.
}

In der Aetiologie der Strumitis haben früher die Affectionen des Digestionsapparates eine bedeutende Rolle gespielt. Ko ch er ${ }^{1}$ ) namentlich erwähnt neben Typhus auch den einfachen Magenkatarrh als ätiologisches Moment. Mit der Zeit hat man schliesslich die Strumitis als mehr oder weniger häufige Begleiterscheinung fast jeder Infectionskrankheit kennen gelernt und in ihrem Eiter beinahe alle Bacterienarten vom Diplococcus Fränkel-W eich selbaum u. s. w. (Tavel) ${ }^{2}$ ) bis zum Pyocyaneus (Lanz und Lüscher) ${ }^{3}$ ) gefunden.

Auch die croupöse Pneumonie spielt eine grosse Rolle in der Aetiologie der Strumitis, und Honsell ${ }^{4}$ ) stellt aus der Litteratur 9 Fälle von metapneumonischer Strumitis zusammen, welche bacteriologisch untersucht, das Vorhandensein von Pneumonie-Diplokokken ergeben haben. In der älteren Litteratur, z. B. Baumann 1856, werden noch mehrere Fälle erwähnt, bei denen der Zusammenhang mit Pneumonie angenommen, aber selbstrerständlich bakteriologisch nicht bewiesen werden konnte.

Eine genaue Zusammenstellung aller, bisher überhaupt beob-

1) Kocher, Zur Pathologie und Therapie des Kropfes. Ueber Entzïndung des Kropfes, zugleich ein Beitrag zur Aetiologie der Entzündung überhaupt. Deutsche Zeitschrift f. Chirurgie. X. S. 191.

2) Tavel, Ueber die Aetiologie der Strumitis. Basel 1892.

3) Correspondenzblatt für Schweizer Aerzte. XXVIII. o. 1898.

4) Honsell, Metapneumonische Strumitis. Beiträge zur klinischen Chirurgie. XX. 1898. 3. S. 601 . 
achteter Fälle von Strumitis findet sich bei Kohn 1), während Hon sell (l. c.) die nach Pneumonie aufgetretenen Strumitiden gesammelt hat.

Wenn auch das Wesentliche über Strumitis hinreichend aufgeklärt ist, so dürfte doch noch jeder neue Fall von Interesse sein.

In Folgendem gebe ich zunächst einen Auszug aus der Krankengeschichte eines in der Zeit rom 12.-22. December 1900 in der chirurgischen Klinik des Krankenhauses im Friedrichshain beobachteten Falles, um dann weiter Bemerkungen darïber anzuschliessen.

„Der ans Posen gebürtige, 52 Jahre alte Barbier F. J. soll aus gesunder Familie stammen und bis etwa 1888 nicht ernstlich krank gewesen sein. Damals will er eine geringe, allmählige Zunahme des Halsumfanges daran gemerkt haben, dass ihm wiederholt der Kragen zu eng wurde.

Seit 1894 hätten sich öfters Scbwindel und Kopfschmerz eingestellt, die mehrere Tage anhielten und J. in dieser Zeit arbeitsunfähig machten.

1870 will J. ein Ulcus molle geLabt haben. Potus wird negirt.

Am 1. December 1900 erkrankte J. plötzlich aus ilım unbekannter Ursache mit Schüttelfrost, Brustschmerzen und Husten. Sein Hausarzt bestätigte mir, dass es sich damals um eine Pneumonie des recliten Oberlappens gehandelt hätte.

Am 5. December 1900 trat eine mässige, wenig schmerzhafte Zunahme der lange bestehenden Halsanschwellung auf. Der Arzt liess mit Jodkalisalbe einreiben und gab Dr. Struve'sche Thyreoidintabletten, sah aber keinen Erfolg.

Dyspnoe trat nicht ein, doch brachte man J. am 12. December 1900 in's Krankenhaus, um bei etwaigem Erstickungsanfall sofortiger Hilfe sicher zu sein.

Status praesens: 12. Decmber. Mittelgrosser, kräftiger Mann in gutem Ernährungszustand mit reichlichem Fettpolster.

Am Hals eine breite Vorwölbung, die in Höhe des oberen Schildknorpelrandes beginnt, nach unten bis nahe an das Iugulum herabreicht, links bis an den Sterno cleido-mastoideus heranreicht, rechts 1 Querfingerbreite vor demselben aufhört. Diese geschwulstartige Vorwölbung lässt sich auf der Unterlage verschieben, am Rande etwas umgreifen und bewegt sich bei der Atmung auf und ab. Die darüber befindliche Hant zeigt keine Veränderung, ist weich, nicht geröthet und lässt sich überall abheben. Palpation schmerzhaft. Etwa in der Mitte der Vorwölbung, links neben der Luftröhre fühlt man eine harte, knirschende Partie, zu beideu Seiten davon deutliche Fluctuation. Grösster Halsumfang tuber die Höhe der Geschwulst: $46 \mathrm{~cm}$. Rachenorgane blass, nicht geschwollen. Kehlkopf frei von krankhaften Erscheinungen. Stimmbänder schliessen bei der Phonation gat.

Ueber dem Oberlappen der rechten Lunge hinten leichte Schallverkürzung, die vorn nicht feststellbar ist. Ueber Mittel- und Unterlappen Schall heller und demjenigen über der linken Lunge gleich. Athem-

1) Kohn, Ueber Strumitis und Thyreoiditis. Allgemeine Wiener medicinische Zeitung. 30. Jahrg. 1885. Nr. $14 \mathrm{ff.}$ 
geräusch über dem rechten Oberlappen etwas verschärft vesiculär von giemenden und rasselnden Geräuschen begleitet, die sich nach dem Mittelund Unterlappen hin immer mehr verlieren. Es besteht Husten und reichlicher, nicht charakteristischer Auswurf.

Athmung mässig beschleunigt, $24 \mathrm{mal}$ in der Minute, regelmässig, frei von Geränschen. Stimme etwas belegt.

Herzdämpfung nicht verbreitert, Herztöne rein. Puls kräftig, etwas beschleunigt 96. Temperatur 38,3. Abdomen frei von krankhaften Erscheinungen.

Urin: sauer, klar, 0 Albumen, 0 Sacharum, 0 Sanguis.

Allgemeinbefinden zufriedenstellend. Am 13. December tritt keine Veränderung ein. Am 14. sind an Hals und Lungen keine objectiv nachweisbaren Veränderungen eingetreten, doch der Puls ist etwas mehr beschleunigt, 116; die Temperatur steigt auf 39,5 , und J. klagt über Schwächegefühl. Die Athmung ist nicht behindert und nur unwesentlich beschleunigt.

In der Nacht zum 15. December treten grosse Unruhe und Delirien auf. Am Tage ist J. fast ganz klar, klagt über grosse Schmerzen am Hals und fublt sich sehr matt. Der Urin ist frei von pathologischen Bestandtheilen, sauer.

Nunmehr wird nach gründlicher Desinfection eine Punction der Halsschwellung vorgenommen, die Eiter ergiebt. Darauf Incision parallel zum linken Sterno-cleido-mastoideus, links von der Trachea. Es entleert sich eine Menge dicken, gelben Eiters mit Kalkstücken bis zu $1 \mathrm{ccm}$ gross und Granulationsgewebe vermischt. Die Eiterhöhle ist mit zahlreichen, spitzen Kalktheilchen ansgekleidet, sie reicht bis unter die linke Clavicula herab, wo man die Arteria subclavia pulsiren fühlt, rechts geht sie nur wenig über die Mittellinie hinaus, während sie links bis an den Sterno-cleidomastoideus heranreicht. Ausspulung mit sterilem Wasser, Ausstopfen mit Jodoformgaze, aseptischer Verband.

Gegen 9 Uhr Abends 40,6 Temperatur bei beschleunigter Puls- und Athemfrequenz, 124, 36. Grosse Unruhe, Delirien. Es wird ein Verbandwechsel vorgenommen, bei dem die Jodoformgazestreifen gelockert aber nicht entfernt werden.

Am 16. December früh besteht die bohe Temperatur $40^{\circ}$ mit den übrigen Krankheitserscheinungen fort. Dann fällt die Temperatur bis zum Abend auf 38,6. Beim Verbandwechsel werden die Jodoformstreifen entfernt; es besteht nirgends Verhaltung, die Secretion ist gering.

17. December. Nach unruhiger Nacht beträgt die Temperatur 38,8 bei 92 kräftigen, regelmässigen, von Schwirren begleiteten Pulsschlägen.

Am Herzen ubber allen Ostien diastolische Geränsche. Im Urin Eiweiss, Esbach $1 / 4$ pro Mille. Mikroskopisch: verfettete Nieren und Blasenepithelien.

Die Wunde secernirt wenig und zeigt keine Retention.

Eingabe von Inf. Digitalis $1,0 / 200,0$ und Thyreoidintabletten.

Gegen Abend wieder Temperatursteigerung 39,9 mit Schüttelfrost bei kräftigem, wenig beschleunigtem Puls.

18. December. Subjectives Befinden besser wie bisher. Die Lungen- 
erscheinungen sind bedeutend zurïckgegangen. Die Schallabschwächung hat sich aufgehellt, es besteht nur noch spärliches Giemen.

Zunge in ganzer Ausdehnung mässig geschwollen, trocken. Feste Speisen machen Beschwerden. Appetit ist gering. J. klagt uber schlechten Geschmack im Munde.

Am 19. December treten keine Veränderungen ein. Es werden einige, erbsengrosse Kalkstücke aus der im übrigen unveränderten Wunde entfernt.

20. December. Die Lungenerscheinungen sind ganz geschwunden, nur noch stellenweise verschärftes Vesiculärathmen.

Am Herzen dagegen keine Veränderung. Die Temperaturkurve ist ausgesprochen septisch. Gestern Abend wieder Schüttelfrost bei 40,1 Temperatur. J. klagt über grosse Mattigkeit und hat ein vermehrtes Schlafbedürfniss. Beim Verbandwechsel wird ein $3 \mathrm{~cm}$ langer, $2 \mathrm{~cm}$ breiter Gewebsfetzen entfernt.

21. December. Eine Veränderung tritt tagstuber niclst ein. Gegen Abend wird der Puls schwächer, Schwächegefühl nimmt trotz Kampferinjection zu und Nachts 2 Uhr erfolgt der Exitus.

Sectionsdiagnose: Abscessus glandulae thyreoideae. Endocarditis verrucosa maligna aortica. Tumor renis sinistri. Infarctus anaemici renis sinistri. Hyperplasis pulpae lienis.

An dem klinischen Verlauf des obigen Falles interessirt zunächst die Erscheinung, dass der Temperaturabfall nach der Operation kein ausgesprochener war, wie sich das in den von Tavel (l. c.) veröffentlichten Temperaturkurven stets fand. In unserem Falle sank die Temperatur bis zum Abend des auf die Operation folgenden Tages allmählig bis 38,6, blieb aber dann am Tage darauf etwa auf gleicher Höhe, um spät Abends schon wieder anzusteigen. Es fand sich keine genügende Erklärung hierfür. Die Wunde secernirte wenig, zeigte keine Retention oder schwere, äussere Veränderung. Die Lungenerscheinungen hatten sich seit Beginn der Krankenhausbehandlung. erheblich gebessert, sodass der Lungenbefund das Fieber auch nicht erklärte. An den übrigen Organen liess sich überhaupt nichts Krankhaftes nachweisen. Deshalb glaubte man, da in vorliegendem Fall die ganze Thyreoidea zerstört oder doch schwer verändert war, das fortbestehende Fieber eventuell als Ausfallserscheinung der Schilddrüsenfunction ansehen zu müssen, wie sie nach Kropfoperationen öfters beobachtet und von Reinbach 1 ) beschrieben worden ist. Man gab daher Thyreoidintabletten in steigenden Dosen. Es trat kein Zeichen ein, das für die Richtigkeit dieser Annahme gesprochen hätte. Da die nächsten Tage schwer septische Allgemeinerscheinungen

1) Reinbach, Ueber das Verhalten der Temperatur nach Kropfoperationen; Mittheilungen ans den Grenzgebieten der Medicin und Chirurgie. 1599. Heft 4. Nr. XX. 
brachten, diurfte auch die trotz Operation hoch bleibende Temperatur als Infectionsfieber anzusehen sein.

Solche Allgemeininfectionen sind nach Strumitis zwar nicht ungewöhnlich, aber bisher doch ziemlich selten beobachtet worden. Oulmont ${ }^{1}$ ) berichtet über einen Fall von pyämischer Infection bei einer 26 jährigen, die von der entzündeten und vereiterten Struma ausging und unter Schüttelfrost, Diarrhoe und Vereiterung mehrerer Gelenke mit dem Tode endete. Im Anschluss an Pneumonie namentlich stellte Honsell (l. c.) bisher nur einen gutartigen Verlauf der Strumitis fest und erklärt das mit der im Allgemeinen geringen Virulenz der in der Struma gefundenen Pneumokokken. Wenn er trotzdem die Prognose zweifelhaft stellt, müssen wir ihm darin beipflichten. Aber die von ihm erwähnte Kompressions- und Senkungsgefahr giebt, wie wir in Folgendem sehen werden, nicht allein eine Verschlechterung: der Prognose. Gerade Erscheinungen dieser Art - und damit komme ich auf eine weitere Eigenthümlichkeit meines Falles - fehlten fast ganz. Ein für den kräftigen Körperbau des Mannes kaum abnormer Halsumfang von $46 \mathrm{~cm}$ an der dicksten Stelle liess nur auf eine kleine Struma schliessen, die an und für sich nur geringe Kompressionserscheinungen hätte machen können. Damit stimmt denn auch die Thatsache ïberein, dass Patient weder vor noch in den ersten Tagen der Krankenhausbehandlung Dyspnoe hatte. Sie trat zu Ende des Krankenlagers auf.

Auch andere Kompressionserseheinungen waren kaum ausgeprägt, wenn man nicht die bei normalem Kehlkopfbefund etwas belegte Stimme dafür ansehen will. Doch auch hierin konnte es sich nicht um eine akute Kompressionserscheinung handeln; denn der Hausarzt gab an, dass die Stimme des J. bereits seit Jahren dieselbe, eigenthümliche Klangfarbe gehabt hat.

Lion Bensaude ${ }^{2}$ ) erwähnt auch einen Fall von Strumitis nach croupöser Pneumonie, der ohne Dyspnoe einberging. Honsell (l. c.) fand degegen in beinahe allen Fällen von Strumitis nach croupöser Pneumonie hochgradige Athembeschwerden. Es kommen demnach beide Erscheinungen vor. Das jeweilige Vortreten der Dyspnoe ist offenbar abhängig von der ursprünglichen Grösse der Struma und von plötzlich einsetzenden Zuständen entzündlicher Schwellung, die auch fehlen können.

Die merkwürdigste Erscheinung bildete das Verhalten der über der Halsanschwellung befindlichen Haut, welche nicht die geringste

1) Oulmont, La France médicale. No. 68. 1880.

2) Lion-Bensaude, Bull. de la soc. anat. de Paris. 1891. 
Veränderung zeigte. Die in der Struma nachweisbare, deutliche Fluctuation liess auf eine reichliche Flüssigkeitsansammlung schliessen. Bei der völlig intakten Haut und weichen Umgebung der Geschwulst konnte man aber zunächst nicht an entzündliche Vorgänge in der Struma denken, sondern wurde mehr auf das Bestehen einer Cyste hingeführt. Nur durch das hohe Fieber in Verbindung mit der zunehmenden Schmerzhaftigkeit der Anschwellung kam man zu richtiger Würdigung derselben.

Die Operation zeigte, dass der Eiter dem deutlichen Fluctuationsgefühl entsprechend ziemlich bis an die Oberfläche der Geschwulst reichte und reichlich war. Um so bemerkenswerther ist es, dass trotz dieser schweren, bis an die Oberfläche reichenden Veränderungen der Struma die äussere Haut noch nicht an dem Process mit betheiligt war, und sich nicht einmal Infiltration derselben nachweisen liess.

In der Litteratur ist bei Schilderung ähnlicher Vorgänge mindestens von Röthung und Schwellung der Haut die Rede. So giebt K ohn (l.c.) unter den Kriterien der acuten Strumitis, um die es sich auch in unserem Fall handelte, eine gespannte, geröthete, sich heiss anfühlende Haut an.

Unter mehrfachem Schüttelfrost, erhöhter Puls- und Athemfrequenz entwickelte sich das Bild einer Sepsis immer deutlicher, waren doch die plötzlich einsetzenden Herzgeräusche mit gleichzeitig auftretender Nierenaffection nur unter der Annahme einer Allgemeininfection zu verstehen.

Ueber die Ursache der schweren Septicämie klärte die bakteriologische Untersuchung auf. Neben zahlreichen Fränkel'schen Diplokokken fanden sich Streptokokken in Eiter und Cultur. Damit war zugleich ein Fingerzeig für die Aetiologie der Erkrankung gegeben. Eine abklingende Pneumonie war vorhanden. Auf ihrem Boden entwickelte sich alsbald die Eiterung in der von früher her bereits veränderten Schilddrüse.

Während die normale Schilddrüse nur in den seltensten Fällen von derartigen Processen ergriffen wird, wissen wir durch Kocher (l. c.), dass es die krankhaft veränderte oder in regressiver Metamorphose befindliche, hyperplastische Schilddrüse ist, welche $\mathrm{zu}$ infectiösen Processen neigt.

Eine primäre Vereiterung nach Trauma, Punktion oder Jodinjection ist in unserem Falle mit Sicherheit auszuschliessen. Patient selbst gab auf Befragen nichts darüber an.

Auf Grund des Befundes handelt es sich im beschriebenen Falle offenbar um eine Struma hyperplastica mässigen Grades, in der aus- 
giebige Vorgänge der regressiven Metanorphose sich abgespielt hatten. Für letztere ist das Vorhandensein einer grösseren Menge kohlensauren Kalkes beweiskräftig. In die Wand der Abscesshöhle waren zahllose, kleine Kalktheilchen eingesprengt, und ein ca. 1 ccm grosses Kalkstück fand sich mitten im Granulationsgewebe.

Das mikroskopische Bild liess eine grösstentheils hyaline Entartung der Schilddrüse erkennen. Neben reichlicher Proliferation von Rundzellen fanden sich Vakuolen, deren Umgebung ein zellarmes Gewebe bildete.

Demnach hat man es hier nicht mit einer entzündlichen Schwellung der bis dahin normalen Schilddrüse, einer Thyreoiditis, sondern mit der Entzündung und Vereiterung einer bereits strumös veränderten Thyreoidea, also einer eitrigen Strumitis zu thun.

Von Bedeutung ist ferner die mikroskopisch festgestellte Thrombose zahlreicher Venen im Strumagebiet, welche an den makroskopisch zu beobachtenden Halsgefässen bei der Section nicht gefunden wurde.

Kocher (1. c.) betrachtet gerade diese Thrombose in der hyperplastischen Schilddrüse als eine der häufigsten Prädispositionen für spätere, eitrige Erkrankung der Drüse. Das Vorhandensein dieser Thromben lässt darauf sehliessen, dass sie der Träger der Infection gewesen sind, und dass die Strumitis als Ausgangspunkt für die Allgemeininfection betrachtet werden kann; denn abgesehen davon, dass die schweren Allgemeinerscheinungen erst nach Abheilung der Pneumonie und mehrtägigem Bestehen der Strumitis auftraten, fand sich in der linken Niere ein anämischer Infarkt, der wohl mit ziemlicher Sicherheit den in der Struma mikroskopisch nachgewiesenen Thromben seine Entstehung verdankt. Ob die vom 18. Dec. ab beobachtete, fortschreitende Glossitis ebenfalls auf thrombotischer Basis beruhte, oder auf isolirte Fortleitung des Entzündungsprocesses von der afficirten Schilddrüse aus zurückzuführen war, ist nicht festgestellt. Immerhin wäre eine solche, isolirte Fortleitung in die Zunge ohne gleichzeitige Betheiligung des Rachens, Kehlkopfes und ihrer Schleimhäute schwer zu erklären, daher muss man wahrscheinlich auch für diese Affection thrombotische, bezugsweise embolische Processe verantwortlich machen.

In der Anordnung der Krankheitserscheinungen ist bisher meist ein unserem Falle entgegengesetztes Verhalten beobachtet worden, da sich gewöhnlich auf dem Boden einer Pyaemie erst eine metastatische Vereiterung der Thyreoidea herausbildete. Wir haben uns also im beschriebenen Falle den Vorgang so zu denken, dass die Strumitis zwar eine metastatische, von der Pneumonie aus entstandene gewesen ist, dass nun aber von ihr die Uebersehwemmung des Körpers mit 
den Erregern der Sepsis stattfand. Meist bildet die Strumitis gewissermassen einen Abschluss der ursprïnglichen Erkrankung, Typhus, Gelenkrheumatismus etc. und ist dann als gleichwerthig mit den nach Infectionskrankheiten lange bekannten, metastatischen Vereiterungen drüsiger Organe aufzufassen. Dann pflegt sie, wie die meisten dieser Drüsenvereiterungen gutartig zu sein und zur Heilung zu führen, wenn nicht etwa die bereits bestehende, abnorme Grösse der Struma durch den Entzündungsprocess zu einem unüberwindlichen, mechanischen Hinderniss für die Athmung wird.

In diesen leichten Fällen handelt es sich wahrscheinlich immer um eine umschriebene Herderkrankung in der Schilddrüse. Es ist vielleicht eine alte Cyste vereitert, an deren Wand dann der Process Halt macht. Daraus würde sich das gutartige Verhalten einer Strumitis mit eitriger Einschmelzung, in der man dann wahrscheinlich nur eine Art von Eitererregern mit geringer Virulenz findet, erklären lassen. Auch die allmählige Entstehungsweise und das oft beobachtete, späte Auftreten solcher Strumitiden würde damit in Einklang zu bringen sein.

Bleibt dagegen der Entzündungsprocess nicht beschränkt, sondern geht diffus in das ganze Gewebe der Schilddrüse unter völliger Zerstörung derselben über, so sind damit die Vorbedingungen für eine septische Allgemeininfection gegeben. Diese Processe treten wahrscheinlich bald nach Beginn der Grundkrankheit auf und spielen sich schnell ab entweder in Folge hoher Virulenz eines Eitererregers oder vielleicht stets auf Grund einer Mischinfection.

Unter diesen Gesichtspunkt würde unser Fall zu betrachten sein. Wir fanden hier eine schwere Schädigung der ganzen Schilddrïse, die durch nekrotischen Zerfall des ganzen Organes gekennzeichnet wurde. Die Vereiterung war eine diffuse. Die Allgemeininfection trat schnell ein. Wie heftig dieselbe war, liess sich aus der bei der Section von Herrn Professor von Hansemann festgestellten Endocarditis verrucosa und der schweren Nierenaffection erkennen.

Während Kohn (l. c.) Fälle von Sepsis nach acuter Strumitis erwähnt, findet sich darunter entsprechend den bisherigen Veröffentlichungen Hon sell's keine pneumonische Aetiologie. Das würde des Letzteren Theorie von der Virulenzverminderung der Pneumokokken stützen. Dieses Beobachtungsergebniss dürfte aber nur als zufälliges aufzufassen sein, da bekanntlich maligne Endocarditis ebensogut durch Pneumo- wie Streptokokken verursacht werden kann.

Dass unser Patient auf die Dauer der Erkrankung nicht Stand halten konnte, ist bei dem Angriff des durch die Pneumonie bereits Doutsche Zeitschrift f. Chirurgie. LIX. Bd. 
geschwächten Körpers durch zwei so hochgradig pathogene Bacterienformen ziemlich selbstverständlich.

Durch diese Möglichkeit allgemeiner, septischer Erkrankung nach der auf pneumonischer Basis beruhenden Strumitis wird die Prognose dieser speciellen Form noch bei weitem ernster. Trotz der meistbeobachteten Gutartigkeit dieser Form wird man auch hierbei die Gefahr eines tödtlichen Ausganges stets im Auge behalten müssen.

Das Ergebniss dieser Arbeit lässt sich folgendermassen zusammenfassen:

1. Die Prognose lässt sich vor operativer Eröffnung der Anschwellung nicht sicher stellen.

2. Nachweis von Streptokokken im Eiter oder derjenige einer Mischinfection giebt stets eine ungünstige Prognose, während jede andere, allein auftretende Bacterienart günstiger zu beurtheilen ist.

3. Die spät auftretenden, metastatischen Strumitiden geben eine günstigere Prognose, wie die schnell einsetzenden. 